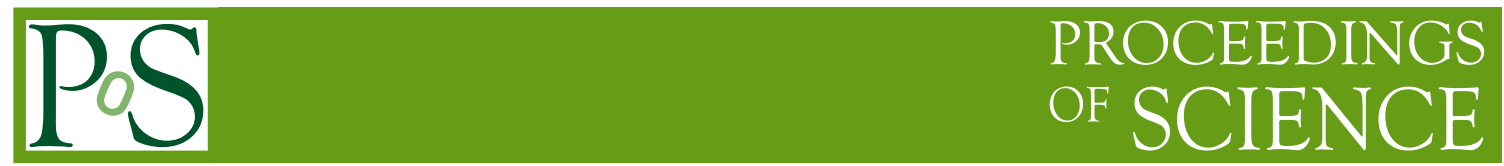

\title{
Dijet physics with the CMS detector at LHC
}

\author{
Cosmin Dragoiu*i \\ University of Illinois at Chicago \\ E-mail: cosmin.dragoiudcern.ch
}

\begin{abstract}
We present preliminary results from the CMS experiment for various dijet distributions in protonproton collisions at a center-of-mass energy of $7 \mathrm{TeV}$. Early measurements of the dijet mass spectra, centrality ratio, azimuthal decorrelation and angular distribution are shown. Prospects for observing evidence for new physics in these distributions is also presented.
\end{abstract}

35th International Conference of High Energy Physics

July 22-28, 2010

Paris, France

\footnotetext{
*Speaker.

${ }^{\dagger}$ for the CMS colaboration
} 


\section{Introduction}

The production of events containing two jets (experimental signature of quarks and gluons) with large transverse momenta (dijets) is one of the basic Quantum Chromodynamics (QCD) processes occurring at hadron colliders. The jets are reconstructed using an anti- $\mathrm{k}_{\mathrm{T}}$ jet clustering algorithm with a distance parameter of 0.5 . The observed jet energy is corrected, on average, for detector effects. The jet energy and position resolutions are currently derived from Monte Carlo (MC) simulations and validated with data to within $10 \%$.

All triggered events are required to have a good primary vertex with $\left|\mathrm{z}_{\mathrm{PV}}\right|<15 \mathrm{~cm}$ and at least five associated tracks. Loose quality criteria are applied to the jets by requiring: the electromagnetic energy fraction $>1 \%$ for $|\eta|<2.6$; less than $90 \%$ of the jet energy in one calorimeter tower and less than $98 \%$ of the jet energy in the hottest Hadronic Calorimeter (HCAL) readout channel.

\section{Tests of Monte Carlo Event Generators}

The dijet azimuthal decorrelation is used to study higher order QCD radiation effects without the need to reconstruct additional jets. Soft-gluon emissions in the initial and final states decorrelate the two leading jets and cause deviations from $\pi$. The measured normalized $\Delta \varphi_{\text {dijet }}$ distributions (Fig. 1) are better described by PYTHIA and HERWIG++ event generators. The MADGRAPH event generator predicts less azimuthal decorrelation than what is observed in data [1].

The comparisons between 2-jet and 3-jet cross sections provide information about the QCD running coupling constant $\alpha_{\mathrm{s}}$. The value of $\mathrm{R}_{32}$ as a function of $\mathrm{H}_{\mathrm{T}}\left(\mathrm{H}_{\mathrm{T}}=\sum \mathrm{p}_{\mathrm{T}}^{\mathrm{jet}}\right)$ is extracted by dividing the inclusive differential 3-jet cross section by the inclusive differential 2-jet cross section. The ratio (Fig. 3) plateaus around 0.8 in agreement with predictions from PYTHIA and MADGRAPH MC calculations [2].

\section{Searches for Physics beyond Standard Model}

The dijet mass spectrum predicted by QCD falls smoothly and steeply with increasing dijet mass. Extensions to the Standard Model (SM) predict massive objects that couple to quarks and gluons giving rise to resonances in the dijet mass spectrum. The measured dijet mass spectrum (Fig. 4) is in agreement with SM predictions. Mass limits on a few models of dijet resonances are set, including a mass limit of $1.67 \mathrm{TeV}$ on string resonances which exceeds previous limits [3].

The dijet angular distributions probe the properties of parton-parton scattering without strong dependence on the details of the parton distribution functions (PDF). The dijet angular distribution is expressed in terms of $\chi_{\text {dijet }}=\exp \left(\left|\mathrm{y}_{1}-\mathrm{y}_{2}\right|\right)$, where $\mathrm{y}_{1}$ and $\mathrm{y}_{2}$ are the rapidities of the two leading jets. In this variable, QCD exhibits a flat distribution, while signatures of new physics (e.g., contact interactions) are expected to produce an excess at low values of $\chi_{\text {dijet }}$. The measured $\chi_{\text {dijet }}$ distributions (Fig. 2) are in agreement with next-to-leading order (NLO) QCD calculations [1].

The QCD processes produce more dijet events at forward rapidities, while the production of new heavy particles, beyond the SM, populates more central rapidities. The dijet centrality ratio, defined as the number of events with both jets with $|\eta|<0.7$ divided by the number of events with both jets with $0.7<|\eta|<1.3$, is sensitive to these effects. The measured dijet centrality ratio (Fig. 5) is flat as predicted by QCD [4]. 


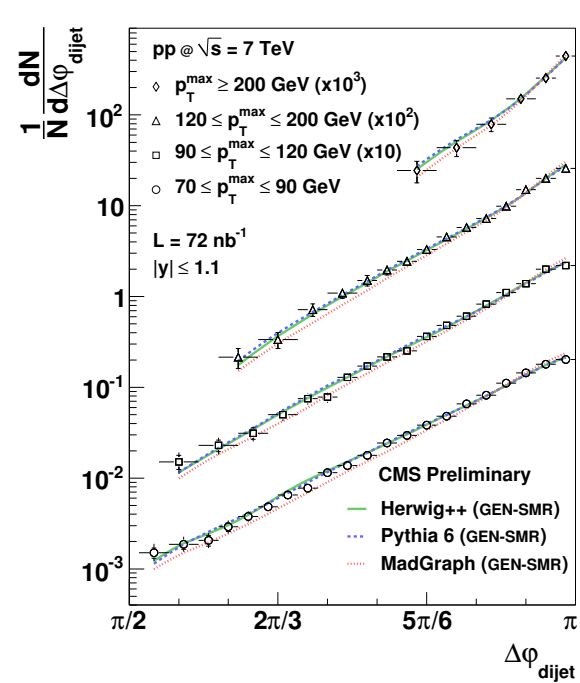

Figure 1: $\Delta \varphi_{\text {dijet }}$ distributions in different leading jet $\mathrm{p}_{\mathrm{T}}$ regions. MC predictions include detector effects. Data points include statistical and systematic uncertainties.
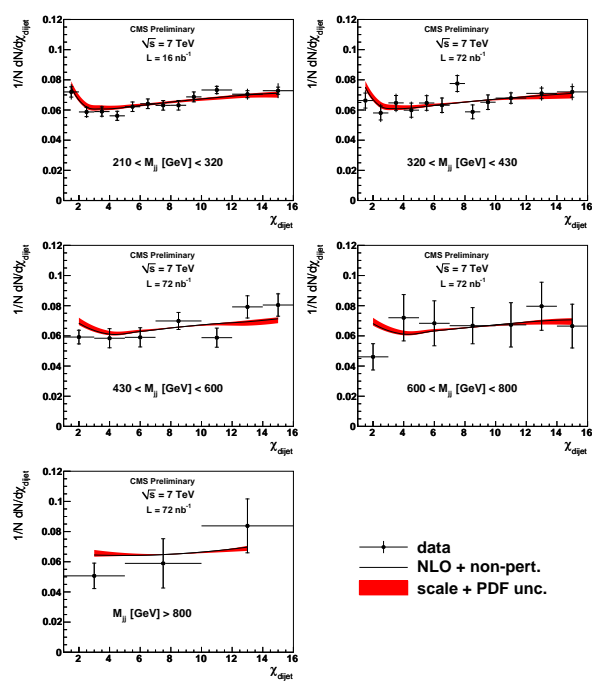

Figure 2: $\chi_{\text {dijet }}$ distributions in different dijet invariant mass regions. Data points include statistical and systematic uncertainties. CTEQ6.6 PDF uncertainties are used.
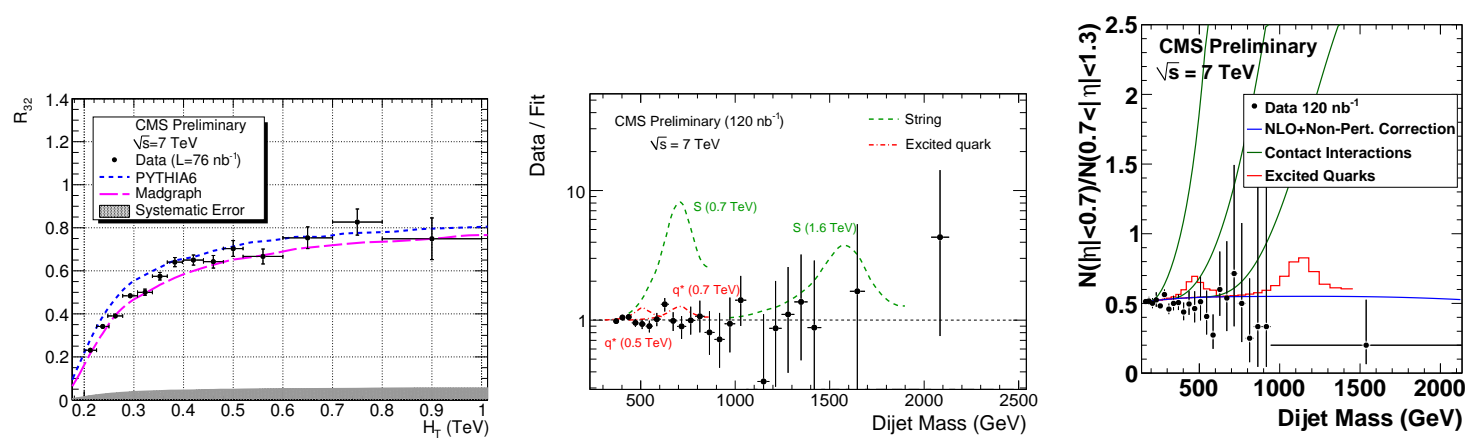

Figure 3: $\mathrm{R}_{32}$ distributions from Figure 4: Ratios between the dijet Figure 5: Dijet centrality radata compared to PYTHIA and mass distribution and a smooth back- tio compared to contact interMADGRAPH. Systematic errors ground fit compared to MC excited actions $(\Lambda=0.5,1.0,1.5 \mathrm{TeV})$ are pictured by the shaded area. quark and string resonance signals.

and excited quark resonances $(\mathrm{M}=0.5,1.2 \mathrm{TeV})$.

\section{References}

[1] CMS Collaboration, Dijet Azimuthal Decorrelations and Angular Distributions in pp Collisions at $\sqrt{s}=7 \mathrm{TeV}$, CMS PAS QCD-10-015 (2010)

[2] CMS Collaboration, Measurement of the 3-jet to 2-jet Cross Section Ratio in pp Collisions at $\sqrt{s}=7 \mathrm{TeV}$, CMS PAS QCD-10-012 (2010)

[3] CMS Collaboration, Search for Dijet Resonances in the Dijet Mass Distribution in pp Collisions at $\sqrt{s}=7 \mathrm{TeV}$, CMS PAS EXO-10-001 (2010)

[4] CMS Collaboration, Search for New Physics with the Dijet Centrality Ratio, CMS PAS EXO-10-002 (2010) 